\title{
Management of paediatric procedure-related cancer pain
} Christina Liossi

\author{
Athens Medical Centre, Athens, Greece
}

\begin{abstract}
Paediatric malignancy is not always painful in its own right; however, young patients with cancer undergo numerous painful procedures for diagnosis, therapy and supportive care, including limbar puncture, bone marrow aspiration and biopsy. Children with cancer consider painful procedures to be the most difficult part of their illness and the frequent repetition of procedures does not desensitize them to the distress. This review provides a brief overview of the state of the art with regard to procedure-related pain in children, and presents some methods and strategies for assessing it and managing it effectively. The first section briefly identifies the dimensions of procedure-related pain and describes the most commonly used methods for its assessment. This followed by an examination of the pharmacological strategies for pain management, including local anaesthesia, conscious sedation and general anaesthesia. In the next section, psychological interventions for the management of procedure-related pain, such as preparation, cognitive-behavioural therapy and hypnosis, are reviewed. The review concludes with recommendations for clinical practice.
\end{abstract}

\section{Introduction}

Childhood cancers are comparatively rare, affecting one in 600 children before the age of 15 years. Each year in the UK there are around 1300 new childhood cancer patients, an annual incidence rate of one per 10000 children. ${ }^{1}$

The outlook for children with cancer has improved radically since the widespread introduction of combination chemotherapy in the late 1960s and early 1970s. Before that time very few cancers could be treated effectively, ${ }^{1}$ but now at least half are probably cured and for many types the survival rates are much higher. For example, the five-year survival rate for acute lymphoblastic leukaemia, the commonest childhood cancer, has increased seven-fold, from 9\% in 1962 to $70 \%$ for children treated in the period 1983-1985. ${ }^{2}$

Although cancer is not always painful in its own right, these patients undergo numerous

Address for correspondence: Christina Liossi, Department of Psychology, Faculty of Applied Sciences, University of the West of England Bristol, St Malthias Campus, Oldbury Court Road, Fishponds, Bristol BS16 2JP, UK. painful procedures for diagnosis, therapy and supportive care, including lumbar puncture (LP), bone marrow aspiration (BMA) and biopsy. Children with cancer consider painful procedures to be the most difficult part of their illness; frequent repetition of the procedures does not desensitize them to the distress. ${ }^{3,4}$ During the 1990s, a growing body of scientific evidence has suggested that the diagnosis of such a threatening condition, followed by the exhausting procedures imposed by highly technical investigations and treatments, are stressors that are potentially responsible for significant psychological distress, anxiety and depression. They negatively affect personal well-being and social functioning, and may represent an obstacle to treatment compliance. ${ }^{5}$ It is essential, therefore, in all children with cancer, that intervention includes concern for and management of the pain and distress associated with procedures.

Numerous studies have documented a high incidence of pain in adults with cancer. ${ }^{6}$ These data, however, cannot be generalized to children because adults differ in factors such as growth, development and types of malignant diseases. 
Chronic, debilitating pain is not encountered so often in children as in adults because of the nature of childhood malignancy. Adult carcinomas tend to be associated with organ systems (lung, stomach, breast) which can result in severe pain, whereas the most common childhood malignancy is leukaemia, which does not usually involve chronic, intractable, disease-related pain. Therefore, categories developed for types of adult cancer pain do not accurately conceptualize children's cancer pain. In addition, although relatively rare in adults, procedure-related pain forms a separate category as a cause of enormous discomfort and anxiety in children. Pain in children with cancer can be reduced to one or more of four basic aetiologies: cancer related (e.g. pain due to infiltration of the tumour in various organs or tissues), treatment related (e.g. pain as a sideeffect of chemotherapy and radiation), procedure-related (e.g. pain due to venepuncture, LP, BMA or postoperative pain), and pain of other aetiologies. $^{7}$

There are few studies that have carefully addressed the incidence of pain in children with cancer. Miser ${ }^{8}$ reports that $60-70 \%$ of adults with cancer have pain that is directly related to the malignancy, while in children direct pain occurs in only about $25 \%$ of patients. Painful episodes are more likely to be due to therapy and procedures $(50 \%)$. A significant amount of pain $(25 \%$ of patients) is completely unrelated to cancer.

Paediatric cancer patients frequently protest at BMAs and LPs. The anxiety and distress produced in anticipation of these procedures is sometimes so severe that children (and often parents) exhibit symptoms such as nausea, vomiting, insomnia, nightmares and skin rashes. Young children often exhibit their distress behaviourally (kicking, fighting, screaming) to such an extent that physical restraint is necessary and it can be very difficult for medical staff to carry out a procedure. Owing to the traumatic nature of BMA and LP, it may take as long as two to three years for children to 'adjust' to the procedures or to encounter them without extreme distress and trauma. ${ }^{9}$ Zeltzer and LeBaron,,$^{10}$ as the initial part of a study looking at behavioural interventions for procedure-related pain, asked 33 children between the ages of six and 17 years to rate on a visual analogue scale (VAS) from 1 to 5 (1 was no pain and 5 was the most pain imaginable) the amount of pain and anxiety associated with LP and BMA. Their rating for pain during BMA was 4.51; during LP it was 3.7. They rated anxiety during BMA 4.2 and during LP 3.75. It is clear that these common procedures cause an enormous amount of distress in children and, for many, represent the worst part of suffering from cancer.

Katz et al. ${ }^{11}$ and Jay et al. ${ }^{12}$ studied children's responses to painful diagnostic procedures. They found that distress was inversely related to age; the younger the child, the greater the distress demonstrated during the procedure. Jay et al. ${ }^{9}$ found that levels of distress were five times as high in young children, with a dramatic decrease at approximately age six to seven years, a time at which children could intellectually understand the need for these procedures, as well the notion that short-term discomfort might have long-term benefit. Even older children, however, still had significant concerns about these procedures.

The 1990s have represented an incredibly exciting period for pain research in general and paediatric pain research in particular. Major advances have been made in the understanding of pain and in the development and refinement of pain measures, as well as in the use of effective pain control methods. This article gives a brief overview of the state of the art with regard to procedure-related pain in children, and presents some methods and strategies for assessment and effective management.

The first section briefly identifies the dimensions of procedure-related pain and describes the most commonly used methods for its assessment. This is followed by an examination of the pharmacological strategies for pain management. Next, psychological interventions for the management of procedure-related pain are reviewed. Recommendations for clinical practice are provided in the final section.

\section{Procedure-related cancer pain}

Pain is a complex, dynamic, multifaceted, perceptual experience, which incorporates cognitive, emotional and social components that affect how a noxious stimulus is interpreted. The context in which noxious information is processed, the cog- 
nitive beliefs of the individual, and the meanings attributed to the presence of noxious sensation are recognized as important factors in shaping the overall experience of pain. ${ }^{13}$ A schematic representation of how the experience of pain may interact with cognitive, emotional, behavioural and physiological variables is presented in Figure 1. Psychological factors are of prime importance in the evaluation of pain. The inexperienced physician has a tendency to overemphasize the contribution of physical factors in individuals, whereas psychologists are more inclined to downplay the extent of the physical factors. Because of the fact that pain is a multifaceted phenomenon, it is important to assess and control it using a multimodal approach targeted at each of the factors that affect the sensory experience.

\section{Assessment and measurement of procedure-related pain}

The distinction between measurement and assessment in pain research is not always clearly drawn. Measurement refers to the application of a scale to a specific aspect, usually intensity, of pain. Assessment is a much broader endeavour, which incudes the identification and detailed evaluation of the different factors of the total experience of pain and their dynamic intereactions. ${ }^{14}$

The importance of pain assessment and measurement in children is based on the premise that, if pain is not assessed regularly and accurately, children will continue to suffer inadequate pain relief. Comprehensive clinical assessment is an absolute prerequisite for successful treatment. The primary purpose of assessment is to design and guide the treatment process. Thus, assessement strategies should not be restricted to pretreatment screening, but, rather, they are a continuous and integral part of the treatment process. They are used to identify and target problems for intervention, to match treatments to the patient's needs and to evaluate the effectiveness of ongoing treatment. Pain measurement in children is complicated by their low verbal fluency and by their changing developmental stages. Despite the problems, however, several pain measurement methods have been reported in the literature. These can be grouped into self-report, behavioural and physiological measures. ${ }^{14} \mathrm{~A}$ number of these methods have been extensively validated, while others are still in the development phase. A full description of all instruments

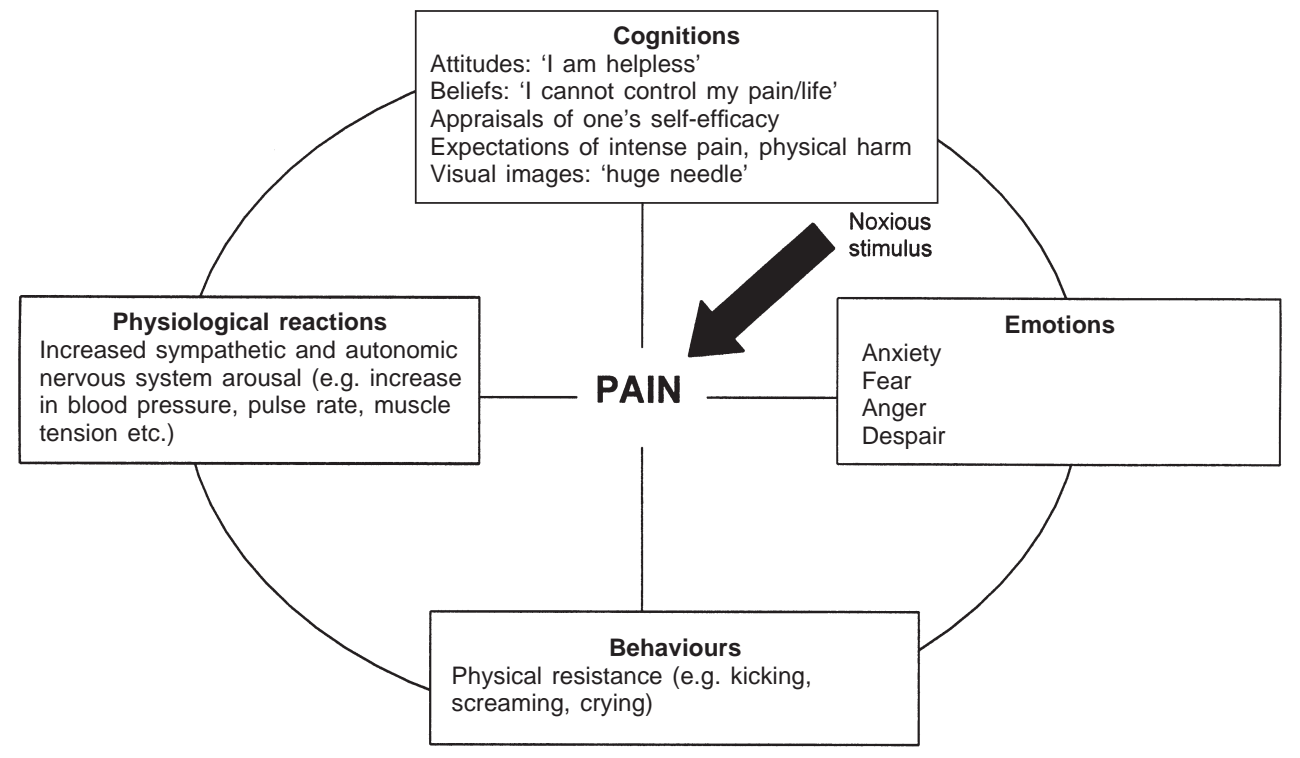

Figure 1 A model depicting the various factors that shape children's perception of procedure-related pain 
is beyond the scope of this article. An attempt will be made, however, to highlight the tools most commonly used in research and clinical practice with procedure-related pain.

\section{Self-report measures}

Self-report measures depend on the child's own report of his or her subjective pain experience. This can include a description of painrelevant feelings, statements and images, as well as information about the quality, intensity and temporal/spatial dimensions of the child's pain. Self-report measures are regarded as the 'gold standard'. However, there are two major problems. First, they require the child to have a level of cognitive and linguistic development, which excludes all preverbal children and may exclude many other young children. The second problem is that self-report measures are open to bias because of the demand characteristics of the specific situation. The methods used to measure selfreport of pain include: direct questioning, pain adjective descriptors, self-rating scales, numerical rating scales and nonverbal methods.

Spontaneous reports (e.g. 'My back hurts') or direct questioning about pain can be useful with verbal preschool and school-age children but, because of their unstructured nature and the lack of an associated measure, they are open to bias because of demand characteristics, inaccurate memory, etc. ${ }^{14}$ Pain adjective lists, such as the McGill Pain Questionnaire, ${ }^{15}$ have been used successfully with older adolescents to measure the sensory, affective and evaluative dimensions of pain. A major strength of this type of scale is that it is not restricted to the intensity dimension of pain. Self-rating scales can be further categorized into three types. A VAS consists of either a vertical or a horizontal line with verbal or pictorial anchors indicating a continuum of no pain to severe pain. Children are asked to indicate on the line how much pain they are experiencing. Category scales consist of a series of words along a continuum in increasing value (e.g. no pain, mild pain, medium pain, severe pain). Faces scales, another form of category scale, consist of pictures of faces expressing varying amounts of distress. Each face is assigned a numerical value reflecting its order within a series of facial expressions. Several variants of face scales ${ }^{16,17}$ have been developed to measure children's level of pain. Numerical rating scales use numbers (i.e. 0-5, 0-10, 0-100) to reflect increasing degrees of pain. Pain thermometers consist of a vertical numerical rating scale with ranges $0-10$ or $0-100$ superimposed on a VAS. The child is asked to point to the place on the thermometer that represents the intensity of pain.

\section{Behavioural measures}

Behaviour observation procedures approach the problem of pain assessment from the vantage point of behavioural indices of pain rather than pain per se and so serve as useful adjuncts to the self-report procedures, physiological measures and documented tissue trauma data available to the clinician or researcher. ${ }^{18}$ Behavioural pain scales record how children respond physically when they experience pain. A trained observer observes the child and records behaviours that suggest discomfort (e.g. crying). Often, behaviours are assigned numbers to represent different intensities of distress.

Three behaviour rating scales have been developed to measure distress in paediatric oncology patients due to BMA and LP: the Procedural Behavior Rating Scale (PBRS), ${ }^{11}$ the Procedure Behavior Check List (PBCL) ${ }^{19}$ and the Observational Scale of Behavioral Distress (OSBD). ${ }^{20}$ Behaviours for observation include crying, screaming, physical restraint, verbal resistance, requests for emotional support, muscular rigidity, verbal pain expression, flailing, nervous behaviour, and information seeking. The developers of all three scales believe that anxiety and pain cannot be separated in these children's behaviour. No distinction can be made between behaviour that is primarily anxiety related and pain-specific behaviour. This position has been criticized because it does not distinguish between, for example, the anticipatory crying of a child in the waiting room, which almost certainly reflects anxiety, and the crying during the needle procedure, which is because of pain. Although anxiety exacerbates the pain experience and pain causes anxiety, the distinction between these constructs is imperative from both theoretical and clinical perspectives. Other disadvantages of behavioural checklists include that: behavioural expressions of pain reflect a number of individual differences 
that are presumed to indicate distress, but may be adaptive coping responses for some children; adolescents usually display fewer overt pain responses; and observers' characteristics, such as experience and attitude, can affect observation scores. $^{19}$

Procedural Behavior Rating Scale Katz et al. ${ }^{11}$ developed and subsequently revised the PBRS. It consists of 13 operationally defined behaviours that are indicative of the distress that young oncology patients exhibit prior to, during and after BMA. Behaviours are coded by observers for occurrence during discrete phases of medical procedures. The PBRS has an inter-rater reliability of 0.85 , but there are few data on its validity. One problem specific to this scale is that it does not discriminate intensity of upset but, instead, merely documents the occurrence or nonoccurrence of the 13 behaviours during the aversive procedures. This means that a few tears and violent sobbing are given identical ratings, and a child who exhibits a variety of behaviours will be scored higher than one who exhibits only a few. The simplicity of this form of scoring generally yields high inter-rater reliability but at the expense of the quality of the information obtained..$^{18}$

Procedure Behavior Check List On the basis of behaviour observations and extensive interviews with childhood leukaemia sufferers regarding their pain experience, LeBaron and Zeltzer ${ }^{19}$ selected eight behaviours that were identical or similar to those of PBRS to form the PBCL. In this adaptation, operationally-defined behaviours are coded by observers for occurrence during discrete phases of a medical procedure. A major problem with the PBCL is the overlapping of categories: 'physical resistance' often includes 'muscle tension', for example, and 'screaming' sometimes includes 'crying'. Because these behaviours can occur together or independently, inter-rater reliability can become a problem. However, in many studies the inter-rater reliability is satisfactory. ${ }^{21,22}$

Observational Scale of Behavioral Distress The $\mathrm{OSBD}^{20}$ is a model of careful scale development. It is an eight-item modification of the PBRS, which introduces two important refinements into the PBRS procedure. Behaviours are recorded at continuous 15 -second intervals within each of the phases of the procedure (e.g. first three minutes in the treatment room, first cleansing of site and Mizzy Gun, second cleansing and aspiration, removal of the needle and postprocedure recovery) rather than recorded once for occurrence or nonoccurrence over an entire phase. Each of the eight behavioural categories (information seeking, crying, screaming, restraint, verbal resistance, emotional support, verbal pain, and flailing) is weighted according to intensity. For example, 'nervous behaviour' is 1.0, 'verbal resistance' 2.5; and 'scream' 4.0. The reliability and validity of the OSBD are satisfactory. The OSBD is more complex than the PBRS and PBCL, and the information it provides is more precise. ${ }^{20}$

\section{Physiological measures}

A number of physiological indicators have been used to measure pain in children; among them there are sufficient data on heart rate, transcutaneous oxygen, sweating and the stress response, to argue their validity as measures of pain in some circumstances. ${ }^{23}$ However, changes in these parameters reflect a generalized and complex response to stress-inducing stimulation, rather than a specific response to pain intensity.

\section{Summary}

All the aforementioned pain assessement methods can be used alone or in combination. When choosing a method, one should first take into consideration the appropriateness of the measure for the age group, the cognitive level, and the clinical and/or research situation. Secondly, if the measure is valid and reliable, the psychometric properties should be known. Lastly, the clinical and research utility of the measure should be checked, such as length, clarity, ease of use and time needed for its use. ${ }^{13}$

In spite of the importance of pain assessment and measurement, pain intensity measurement instruments such as VASs and faces scales are not used regularly in clinical practice. For instance, in Sweden, in a nationwide survey of the 47 paediatric departments where oncology patients are treated, a VAS to score pain intensity is used in $31 \%$ and some kind of face scale 
is used in $23 \%$. Systematic behavioural observation, regular contact with pain treatment teams and the use of a pain diary are rarely used. ${ }^{7}$

\section{Perception of Procedures Questionnaire}

Remarkably few parent report measures for child distress during medical procedures exist in the literature. The most useful one is the Perception of Procedures Questionnaire (PPQ). ${ }^{24}$ This consists of nineteen seven-point Likert-type questions, with lower scores indicating higher satisfaction and less distress. It is easily administered to parents (completion time $<10$ minutes) and provides data on both child and parent distress. The PPQ assesses the immediate procedural context, while also reflecting parental perceptions of general satisfaction with and involvement in their child's treatment. It has good psychometric properties. Factor analyses yielded five factors for parents: parent satisfaction; child distress during the procedure; child distress before the procedure; parent distress; and parent involvement. Parents are asked about their communication with the health care team; their views on the team's commitment to parental concerns about treatment and related side-effects; the extent to which services are comprehensive and include emotional support; their perceptions of their own involvement; and their view of their child's as well as their own distress.

\section{Management of procedure-related pain in paediatric oncology}

The goal of pain management in paediatrics is to minimize the patient's suffering and permit a successful procedure. However, producing a cooperative child is an insufficient goal if the child suffers 'in silence'. ${ }^{25}$ The management of pain associated with paediatric procedures is not a simple task; there are multiple approaches to and philosophies regarding the treatment of procedure-related pain. Different treatment approaches are practised at various treatment centres, and by individual practitioners within a particular setting. One extreme is the practice of providing no pharmacological sedation and analgesia, based upon the belief that the pain is relatively mild and short lived. The risk of medication is seen as unwarranted and the procedure is viewed as being for the child's benefit, thereby justifying minimum intervention. Under these circumstances, children are often restrained by several adults during the procedures. At the other extreme is the administration of general anaesthesia, emanating from the belief that the pain is very intense and that this facilitates the procedure medically. There are many alternatives to these extremes. The methods cited in the literature to manage paediatric pain are commonly grouped under the headings 'pharmacological' and 'nonpharmacological/psychological'. Although both psychological and pharmacological methods are quite effective, their use in the clinical setting is still hampered by a number of misconceptions, as well as by a lack of knowledge regarding their effectiveness in the treatment of pain in children. The psychological and pharmacological management strategies commonly used to help children to cope with paediatric procedures are reviewed below.

\section{Pharmacological strategies for management of procedure-related pain}

Despite the available psychological measures to control pain and reduce distress, children, nevertheless, require pharmacological assistance in helping them to cope successfully with painful procedures. However, when possible, these children should also be given the benefit of psychological support, even if a pharmacological agent is also to be used. ${ }^{25}$

The needs of the individual and the type of procedure to be performed determine the pharmacological approach required. For all patients, an opioid or a local anaesthetic is needed to reduce the pain. Anxiolytics and sedatives are used specifically to reduce anxiety before and during the procedure but, if used alone (i.e. without an analgesic), they may blunt the behavioural response without relieving the pain. ${ }^{25}$

Local anaesthesia Local anaesthetics may be administered by local infiltration (e.g. into the skin, subcutaneous tissues or periosteum with $1 \%$ procaine or $1 \%$ lidocaine for BMA) or topically. Local anaesthetics are extremely useful for providing anaesthesia and analgesia for infants and children of all ages. Despite some toxicity issues (e.g. convulsions), the overall safety record of their use in paediatrics has been very good, and 
their administration within safe guidelines should be encouraged. ${ }^{26}$

An area that has been investigated more specifically and with positive results is a eutectic mixture of local anaesthetics (EMLA), consisting of lidocaine and prilocaine suspended in an oilin-water emulsion. This mixture contains $50 \%$ of each of the local anaesthetic bases. When EMLA cream is applied to intact skin (the recommended dose is approximately $1.5 \mathrm{~g} / 10 \mathrm{~cm}^{2}$ ) and is covered with an occlusive dressing for 60-120 minutes, anaesthesia develops in the underlying tissue. In all instances, maximal development of analgesia is achieved by 120 minutes. After removal of the cream from the skin, analgesia persists in the underlying tissue for several hours. Adverse effects are limited to mild and transient local skin reactions (i.e. blanching, oedema and erythema), which resolve spontaneously within 1-2 hours after removing the cream. For children and infants older than six months, plasma concentrations are uniformly extremely low and the application of EMLA according to the package insert seems extremely unlikely to produce systemic toxicity. ${ }^{27}$

A number of investigators have evaluated EMLA (see Table 1). In a double-blind, placebocontrolled, cross-over trial ${ }^{28}$ the efficacy of EMLA was tested in 14 children aged between 5.5 and 15.3 years who were undergoing LP in association with intrathecal chemotherapy, with one to six weeks between the respective crossover treatments. EMLA cream was applied for a mean time of 73 minutes. The mean pain scores subsequently obtained were 1.9 with eutectic lidocaine/prilocaine cream and 5.6 with placebo (10-point VAS; $p<0.001)$. Twelve of the 14 children expressed a preference for the eutectic lidocaine/prilocaine cream.

Since this trial, two cross-over trials, one openlabel and one double-blind, have been conducted in children undergoing lumbar puncture. ${ }^{29}$ In the open-label study, which involved 18 patients aged five to 15 years, eutectic lidocaine/prilocaine cream ( $2 \mathrm{ml}$ applied between 45 and 60 minutes prior to LP) was compared with no treatment. The mean pain scores on a five-point VAS were 1.66 with eutectic lidocaine/prilocaine cream and 2.55 with no treatment $(p<0.0005)$ according to the patients' assessment, with similar results from parents' and nurses' ratings. In the double-blind study, 10 patients aged 4.5 to 11 years (mean 6.1) received eutectic lidocaine/prilocaine cream (applied as described above) or placebo. The mean pain scores on the five-point VAS were 2.0 with eutectic lidocaine/prilocaine cream and 3.1 with placebo, as rated by nurses $(p<0.05)$. Scores on a 'facial expression' visual scale were 2.9 and 3.8, respectively (difference not significant).

Finally, Calamandrei et al. ${ }^{30}$ compared EMLA cream plus a vapour-permeable adhesive film dressing (Tegaderm) with a prepackaged EMLA patch, with regard to analgesic effect and local reactions during LP in 24 children aged from three to 16 years, who was suffering from leukaemia or non-Hodgkin's lymphoma. There was no difference between the two groups in the pain and observed distress associated with LP or in terms of overall local reactions. This study was performed in such a way that no conclusion regarding the level of analgesia was possible because no control group was used. However, the low pain scores indicate that effective analgesia was achieved during LP by using the EMLA preparations. The EMLA patch is a major advance because it provides a topical anaesthetic in a unit dose preparation, thus allowing for control of the dose administered per application and therefore preventing over- or underdosing. It is easy to apply, simplifies and speeds up the application of EMLA, and is not messy. It allows the convenient home application of EMLA before the patient visits the hospital, when the waiting time can be spent constructively, thus reducing anticipatory anxiety.

In summary, eutectic lidocaine/prilocaine cream provides a painless, well-tolerated method of local anaesthesia/analgesia for procedures such as vein and lumbar punctures in children. Moreover, in procedures in which it is a suitable alternative to local infiltration anaesthesia, it is likely that patients would prefer EMLA cream to multiple intradermal injections, which can be painful and distressing.

Conscious sedation The use of conscious sedation is highly recommended in the clinical and research literature for the management of pain and distress associated with procedures such as BMA and LP. 
Table 1 Comparison of pharmacological intervention studies utilizing EMLA for paediatric procedure-related cancer pain

\begin{tabular}{|c|c|c|c|c|c|c|}
\hline Study & Patients $(n)$ & Age range (years) & Design & Interventions & Outcome measures & Results \\
\hline Halperin et al. ${ }^{28}$ & 14 & $5.5-15.3$ & Cross-over & $\begin{array}{l}\text { EMLA cream } \\
\text { Placebo cream }\end{array}$ & Self-reported pain & $\begin{array}{l}\text { Significant difference } \\
\text { between EMLA and } \\
\text { the placebo }\end{array}$ \\
\hline Kapelushnik et al. ${ }^{29}$ & 18 & $5-15$ & $\begin{array}{l}\text { Cross-over } \\
\text { open }\end{array}$ & $\begin{array}{l}\text { EMLA cream } \\
\text { No treatment }\end{array}$ & $\begin{array}{l}\text { Self-reported pain } \\
\text { Parent-reported pain } \\
\text { Nurse-reported pain }\end{array}$ & $\begin{array}{l}\text { Significant difference } \\
\text { between EMLA and } \\
\text { no treatment pain } \\
\text { scores of the children, } \\
\text { parents and nurses }\end{array}$ \\
\hline Kapelushnik et al. ${ }^{29}$ & 10 & $4.5-11$ & $\begin{array}{l}\text { Cross-over } \\
\text { double-blind }\end{array}$ & $\begin{array}{l}\text { EMLA cream } \\
\text { Placebo cream }\end{array}$ & $\begin{array}{l}\text { Self-reported pain } \\
\text { Nurse-reported pain }\end{array}$ & $\begin{array}{l}\text { Significant difference } \\
\text { between EMLA and } \\
\text { placebo pain scores } \\
\text { for both the children's } \\
\text { and the nurses' ratings }\end{array}$ \\
\hline Calamandrei et al. ${ }^{30}$ & 24 & $3-16$ & Cross-over & $\begin{array}{l}\text { EMLA patch } \\
\text { EMLA cream } \\
\text { plus a vapour- } \\
\text { permeable } \\
\text { adhesive film } \\
\text { dressing }\end{array}$ & $\begin{array}{l}\text { Self-reported pain } \\
\text { Observer-reported } \\
\text { pain }\end{array}$ & $\begin{array}{l}\text { No differences in } \\
\text { pain and observed } \\
\text { distress scores } \\
\text { between the two } \\
\text { groups }\end{array}$ \\
\hline
\end{tabular}


The Committee on Drugs of the American Academy of Pediatrics defines conscious sedation as:

a medically controlled state of depressed consciousness that allows protective reflexes to be maintained, retains the patient's ability to maintain a patent airway independently and continuously, and permits appropriate response by the patient to physical stimulation or verbal command..$^{31}$

Although there are no absolute contraindications to conscious sedation, children with a known airway problem, apnoea, haemodynamic instability, altered mental status, or a previous adverse reaction to sedation or anaesthesia, are identified as at increased risk for adverse cardiorespiratory effects of conscious sedation. ${ }^{31,32}$

Multiple pharmacological agents are discussed in the conscious sedation literature. A combination of a benzodiazepine (midazolam) and an opioid (either morphine or fentanyl) is most frequently recommended for intravenous conscious sedation. ${ }^{32-37}$ This combination has advantages for procedure-related pain management because it provides both analgesia and sedation effectively and safely, ${ }^{32,36,37}$ has a predictable, rapid onset and a short duration of action, ${ }^{32-35}$ can be administered easily and titrated to individual patient response, and may be reversed with the appropriate antagonists. ${ }^{32,35}$ The benzodiazepine, midazolam, has sedative, anxiolytic and amnestic effects, whereas the opioids, morphine or fentanyl, have an analgesic effect. The recommended dosage ranges provided in the literature ${ }^{32,33,35-37}$ are: for midazolam, $0.05 \mathrm{mg} / \mathrm{kg}$ intravenously, initially up to a maximum total dose of $0.15 \mathrm{mg} / \mathrm{kg}$, diluted to a concentration of $1 \mathrm{mg} / \mathrm{ml}$ with $0.9 \%$ saline; for morphine, $0.05-0.1 \mathrm{mg} / \mathrm{kg}$ intravenously, diluted to $1 \mathrm{mg} / \mathrm{ml}$ with $0.9 \%$ saline; and, for fentanyl, $0.5-1 \mu \mathrm{g} / \mathrm{kg}$ intravenously, undiluted.

The most common serious adverse effect of conscious sedation with this combination is respiratory depression with resultant hypoxaemia. ${ }^{32-34}$ As both benzodiazepines and opioids may produce respiratory depression, their administration in combination may produce an additive effect. ${ }^{32}$ For the prevention of respiratory depression and hypoxaemia, the recommendations are for: a careful dosage calculation with a possible reduction of the dosage of individual agents when given in combination; the titration of doses administrated to effect, allowing adequate time for the agents to take effect before administering additional doses; and continous monitoring of the sedated patient for early indications of cardiorespiratory instability. Reversal agents for conscious sedation are flumazenil, a specific benzodiazepine receptor antagonist, for midazolam, and naloxone, a pure opioid antagonist, for morphine or fentanyl. ${ }^{33,35}$

The necessary monitoring of the patient includes the baseline assessment of heart rate, respiratory rate, blood pressure, skin and nail bed colour, level of consciousness, responsiveness and comfort level before sedation, and frequent (every five minutes during the procedure) assessment of heart rate respiratory rate, and blood pressure after conscious sedation. ${ }^{31}$ Continuous oxygen saturation monitoring is essential from the time of administration of conscious sedation until the child is fully alert after the procedure. ${ }^{31}$ Oxygen, suction and airway management equipment, and emergency drugs and supplies, should all be readily available. It is imperative that medical and/or nursing staff who administer conscious sedation and monitor patients during and after sedation should be trained in advanced paediatric life support. A child has fully recovered after concious sedation if there is a return to the baseline level of alertness, and if he or she has stable cardiovascular function and airway patency.

Clinical research supports the efficacy and safety of conscious sedation with midazolam or fentanyl, or a combination of midazolam and fentanyl or morphine. Sievers et al. ${ }^{33}$ studied 70 procedures (BMAs or biopsies and LPs) performed on 24 ambulatory paediatric oncology patients aged 1-15 years. This study found that midazolam, either alone or in combination with fentanyl or morphine, provided effective sedation as measured by behavioural distress scores and that no restraint was required for $45 \%$ of the procedures. Hypoxaemia, as indicated by arterial oxygen saturation level $\left(\mathrm{SaO}_{2}<90 \%\right)$, occurred in $13 \%$ of the procedures. All occurrences of hypoxaemia resolved with stimulation to respiration and the administration of oxygen by mask. All patients had completely recovered from sedation within 
60 minutes after the last dose of midazolam was administered.

Sandler et al. ${ }^{34}$ studied 86 procedures (BMAs and LPs) performed on 25 ambulatory paediatric oncology patients aged 3-21 years. In a randomized, double-blind, cross-over study comparing midazolam and fentanyl, both agents were found to provide effective sedation as measured primarily by behavioural distress scores and parent/child satisfaction with the conscious sedation experience. Seventy-two per cent of the parent/child preference was for midazolam, which the investigators hypothesized was due to its amnestic effects. Hypoxaemia $\left(\mathrm{SaO}_{2}\right.$ 90-95\%) occurred in two patients who received midazolam and two patients who received fentanyl; it resolved with respiratory stimulation and oxygen administration. No bradycardia or chest wall rigidity was observed in the patients who received fentanyl, although these effects have been reported in other studies.

General anaesthesia Some experts maintain that short-acting general anaesthesia is a highly preferable alternative to premedication for outpatient procedures or surgery. ${ }^{38}$ Short-acting general anaesthesia is administered in the majority of paediatric oncology departments in Europe to children who are undergoing $\mathrm{BMA},{ }^{39}$ but it is used on a much more limited basis in the USA, in only a few of the larger paediatric oncology centres. ${ }^{40,41}$

More specifically, the safety and low incidence of the negative side-effects of halothane have been well documented. The possible complication of post-halothane jaundice and liver toxicity has been investigated in two retrospective studies, ${ }^{42,43}$ which assessed the risks of jaundice associated with halothane to be between one in 82000 and one in 200 000, respectively. Fisher et $a l .{ }^{40}$ assessed the efficacy of halothane compared with two other inhalation anaesthetics (enflurane and isoflurane) for helping children who underwent BMA or LP. In that study of 66 children (aged eight months to 18 years) and 124 procedures, the total time from the start of the procedure to discharge averaged 22.3 minutes for halothane, which was almost identical to the other two agents. However, halothane demonstrated the lowest incidence of excitement and coughing. The incidence of laryngospasm was lower for both halothane and enflurane, and reported as mild in all affected patients.

Although halothane appears to be a safe, reliable and short-acting anaesthetic agent with limited side-effects, more data are needed to determine if this is a viable approach for children undergoing LP and BMA. The obvious disadvantages of general anaesthesia include additional costs, the need to co-ordinate scheduling with the operating theatre and an anaesthetist, and an increased risk of complications. Moreover, even though halothane provides a pain-free procedure, the children and the parents may not be without distress, given that these patients are allowed nothing by mouth for at least eight hours before the procedure, some children find mask induction distressing, and parents may be concerned about the medical risks involved. ${ }^{44}$

A new intravenous agent, propofol, has greatly facilitated intravenous approaches to conscious sedation and anaesthesia because it permits a rapid induction and a clear-headed, euphoric and rapid emergence. Unlike most other anaesthetics, propofol has anti-emetic and antipruritic actions. ${ }^{45}$ However, further research is necessary to determine its safety and clinical usefulness because a recent report indicated the possibility of delayed seizures after propofol anaesthesia. ${ }^{46}$

Clearly, more research is needed to explore further: children's emotional and behavioural responses to general anaesthesia; its acceptability by children, parents and caregivers; the intensity and duration of side-effects; and cost-benefit considerations, particularly in relation to more commonly used psychological interventions. $^{44}$

As should be evident, a number of approaches have been tried, but there is no consensus, either clinically or in the research literature, regarding the use of appropriate pharmacological agents for the management of the pain and anxiety associated with painful medical procedures.

\section{Nonpharmacological interventions for procedure-related pain}

Psychological interventions aim to decrease pain and distress and enhance children's abilities to cope successfully with painful procedures. ${ }^{36}$ This section provides an overview of the inter- 
ventions most commonly used in clinical practice and evaluated in research.

Preparation Preparation is the most common psychological intervention for children who undergo painful medical procedures. It is a generic term for a number of techniques and procedures, the central component of which is provision of information to children and their parents concerning the necessity, nature and various phases of the impending medical procedure. The rationale underlying preparatory interventions is that unexpected stress is more anxiety provoking and more difficult to cope with than anticipated or predictable stress. ${ }^{47}$ Children who undergo systematic preparation, rehearsal and supportive care respond best before, during and after the procedure. Adequate information and communication with the patient are therefore of vital importance.

Preparatory information can be characterized as being sensory or procedural. ${ }^{48}$ Procedural information involves the depiction of the steps of the procedure, without descriptions of sensations. Sensory information involves a description of the sensations the patient will experience at various points in the procedure. For example, a child being prepared for a BMA might be told that the cleansing of the back would feel 'cold', whereas the numbing medicine would feel like a 'pinch' or a 'prick'. The information that is most effective appears to be a combination of sensory and procedural data. ${ }^{49}$

In actual clinical practice, preparation involves the following components: introducing the child to medical personnel; giving the child developmentally appropriate information about what will be done and how it will feel; letting the child handle equipment; having the child practice the procedure on a doll; and discussion of the child's feelings and thoughts about the procedure. Various methods have been used to provide children with information about painful medical procedures, including doll-play, hospital tours, story and colouring books, and cognitive strategies. ${ }^{36}$

Despite wide agreement about the benefits of preparing children for painful procedures, there have been few attempts in the literature to establish clear guidelines about how young patients should be prepared for their treatments. There are three phases in performing a painful medical procedure where adequate preparation is relevant. These phases and a summary of the key steps within each are presented next and also shown schematically in Figure 2. Phase one involves preparation before the procedure and consists of explaining to the patient in a clear, developmentally appropriate language why the procedure is needed, what will happen and how it will feel. The patient must be given the

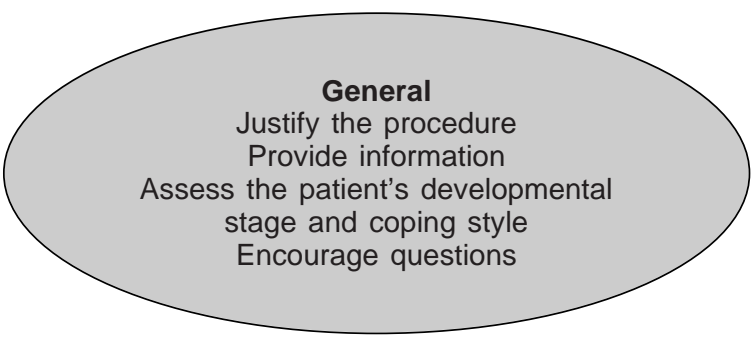

Before the procedure

Provide procedural and sensory information Give the patient specific instructions on what to do during the procedure

Explore and address concerns

Introduce new coping skills

Provide emotional support

Summarize important information at the end

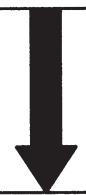

\section{During the procedure}

Provide procedural and sensory information

Give the patient decisional control

Encourage the use of coping skills

Provide emotional support

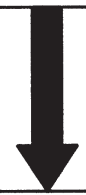
After the procedure
Debrief the patient
Encourage the use of coping skills
Provide emotional support

Figure 2 Steps involved in adequately preparing a child for a painful medical procedure 
opportunity to ask questions and express concerns. An assessment of the patient's coping style can be performed so as to suggest appropriate coping strategies. Phase two involves care during the procedure, when the health professionals can, if the patient desires, explain what is going on and how it is likely to feel, and encourage and reinforce the patient's attempts to practise coping strategies. It is important that children are given decisional control, rather than absolute or behavioural control. With decisional control, the child may be offered, for example, the choice of the procedure beginning in three or seven seconds, rather than after an indefinite delay, as is typical of behavioural control. Giving children limitless control leaves them to their own devices about how to manage themselves in the situation and increases their anxiety. Finally, phase three involves care during recovery. During this phase it is useful to debrief the young patient and encourage him or her to take an active part in recovery efforts. The patient can continue to use coping skills. Further research and controlled clinical trials will be necessary to evaluate which of these steps will be most helpful to individual patients. However, as each patient suffers in his or her own unique way, clinical sensitivity must always take priority over general guidelines.

More specifically, the assessment of children's cognitive coping styles is important in planning interventions and in predicting treatment outcome. Cognitive styles include dimensions such as internal-external locus of control, ${ }^{50}$ representing repression-sensitization, minimization-vigilant focusing, ${ }^{51}$ monitor-distractor, ${ }^{52}$ and approach-avoidance. ${ }^{52}$ Locus of control refers to a person's perception of whether life events are controlled and influenced by oneself (internal) or by external forces such as fate, luck, chance and powerful others. Neuhauser et al. ${ }^{50}$ found that children who have high internal control perceive themselves as having more control over the healing process. Although Jay ${ }^{53}$ found no relationship between locus of control and children's distress levels during painful medical procedures, further research is needed to clarify how children's perceived control over illness and pain relates to coping processes. The three cognitive styles described in the literature (repression-sensitization, minimization-vigilant focusing, and monitor-distractor) all describe a similar construct. People referred to as repressors, minimizers, or distractors are those who tend to use selective inattention, denial, avoidance of information and rationalization when dealing with a stressful event. On the other hand, people referred to as sensitizers, vigilant focusers and monitors are those who cope with stressful events by actively seeking information, focusing on details, and practising coping skills. Although little or no research has investigated these dimensions in children, it is believed that, for optimal intervention outcome, the matching of intervention techniques with individual coping styles is necessary. For instance, patients who avoid anxiety-provoking stimuli should be supported by additional distraction techniques, whereas vigilant patients who spontaneously focus their attention on these stimuli should be shown how to transform them in a positive context.

Cognitive-behavioural intervention Human beings are active processors of information. They deal with information about what is happening around and within them according to their previous experiences, their expectations, appraisals, mental images and beliefs. According to the cognitive model, the individual's interpretation of events - not the events or 'objective reality' as such - influences behaviours and affective reactions. Moreover, how persons evaluate and predict their capabilities to influence a situation, and the probability and likely consequences of achieving this aim, further shape their perception of an event. ${ }^{54}$ The rationale for cognitive therapies is that, because a patient's cognitions determine their stress reactions, modifying negative and maladaptive cognitions is one way to reduce distress. ${ }^{55}$ For pain, the cognitive-behavioural model emphasizes that it is not simply a sensation but a complex, multidimensional experience. Therefore, pain needs to be assessed and treated not only in terms of its sensory intensity but also in terms of its cognitive and affective qualities, and its effects on pain-related behaviours.

Jay et al. ${ }^{56,57}$ developed a multicomponent intervention 'package' of cognitive-behavioural techniques (CBT) designed to teach effective coping skills and to reduce children's distress during BMA and LP. This consists of five primary 
components: filmed modelling, incentive, breathing exercises, emotive imagery and distraction, and behavioural rehearsal. Patients are first shown an 11-minute film of another child undergoing a BMA or LP. In this film, the child narrates the steps of the medical procedure, together with thoughts and feelings at critical points, and models positive coping behaviours (breathing exercises, imagery) and positive 'self-statements' (e.g. 'I know, I can do it') during the medical procedure. Since research supports the superiority of modelling based on coping as opposed to mastery models, the child in the film exhibits a realistic amount of anxiety (e.g. 'I am a little scared'), but then copes effectively, as opposed to not exhibiting anxiety or distress. After the film, patients are taught a simple breathing exercise. Each child is instructed to take a deep breath and to let it out slowly while making a hissing sound ('s-s-s'). This serves as an attention-distraction technique and gives the child something active to do during the procedure, rather than being passively submissive.

The provision of incentive involves showing the child a small trophy, which is presented as a symbol of mastery. Children can obtain the trophy if they do 'the best that they could possibly do'. Each child is encouraged to lie still (which reduces the likelihood of complications in conducting the medical procedure) and carry out their breathing exercises (which generally preclude severe behavioural distress, such as kicking, screaming, etc.). The purpose of the trophy is to try to transform the meaning of pain for the child. Rather than perceiving the LP or BMA as a distressing, punitive event, the idea is to try to encourage children to perceive it as a challenge and as an opportunity to master a difficult situation, hence resulting in positive feelings of selfefficacy and increased self-esteem.

Emotive imagery is a technique that involves ascertaining a child's superhero or fantasy images and weaving these images into the current medical situation. For instance, children may wish to imagine that they are Superman's agent and that Superman has asked them to undergo the medical procedure as part of a special mission. Such images presumably transform the meaning of the pain for a child and provide another distraction technique.
The final component of the package is behavioural rehearsal. In this phase the child is encouraged to 'play doctor' and to give a doll the BMA or LP using actual medical equipment (with older children, it is called a demonstration). The rehearsal provides information to the child and allows him or her mentally and emotionally to prepare for the impending medical procedure.

Jay et al. conducted a series of studies (Table 2) that systematically examined distress behaviour, antecedent variables, and the effects of behavioural and pharmacological interventions on procedural distress. In their first study, ${ }^{56}$ five children aged 3-7 years, referred for severe anxiety and behavioural distress related to BMA or LP, reduced their distress scores by at least $50 \%$ after intervention.

The second study ${ }^{57}$ was a comparison of the CBT package with oral diazepam and an attention-control condition (30 minutes of cartoon watching prior to the BMA), delivered in the context of a repeated measures, counterbalanced design. A total of 56 children with leukaemia, aged 3-13 years, were studied. Dependent variables were scores on the OSBD, self-reported pain, pulse and blood pressure readings. Overall, children had significantly lower pain ratings, OSBD scores and pulse rates when in the CBT condition when compared with either the diazepam or the attention-control group. Diazepam was useful for lowering anticipatory distress but had no effect on distress during the procedure (encounter phase). There was no difference between treatments on OSBD scores when the encounter phase of the BMA was analysed separately.

The next study ${ }^{58}$ investigated the combined effects of oral diazepam and CBT. Within a repeated-measures factorial design, 83 children aged 3.5-12 years were assigned to either a CBT or CBT plus diazepam condition. Dependent variables included observed behavioural distresses measured by the OSBD, self-reported pain and anxiety, and pulse rate. Both groups reduced their OSBD and self-reported pain scores from baseline to intervention; however, the CBT plus diazepam group demonstrated only one-third of the reduction compared with children in the CBT only group. The investigators suggested that the diazepam may have interfered 


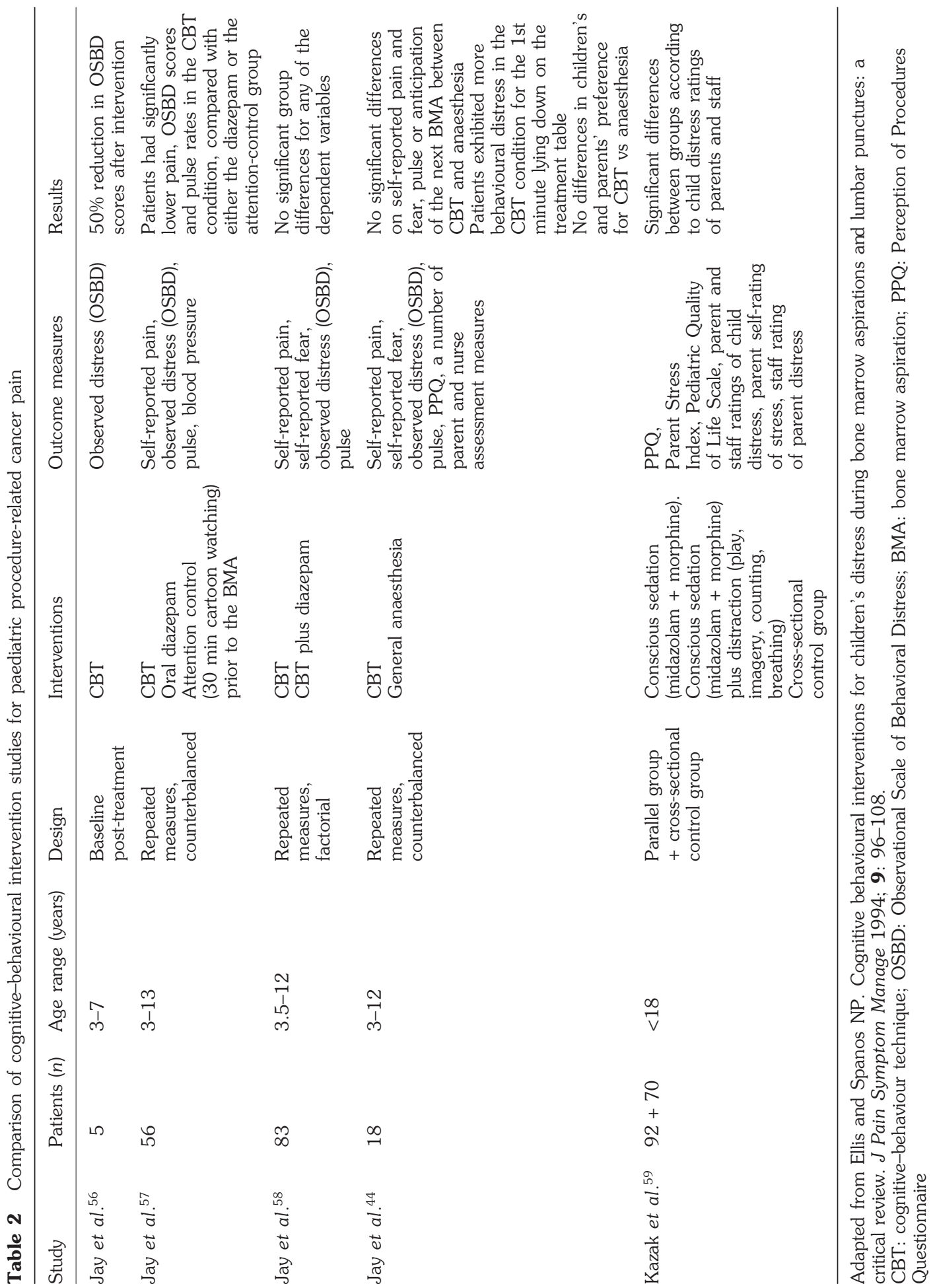


with the learning of the CBT strategies. In addition, the children may have been unable to remain focused on the imagery and distraction tasks during the procedure, owing to the effects of diazepam.

The last study ${ }^{44}$ was a comparison of CBT with general anaesthesia in alleviating the distress of 18 paediatric cancer patients (age 3-12 years) who were undergoing BMA. CBT and short-acting mask anaesthesia were delivered within a repeated measures, counterbalanced design and subjects were assigned randomly to one of two sequence orders. The results indicated that children exhibited more behavioural distress in the CBT condition for the first minute of lying down on the treatment table. With respect to the child outcome measures, no significant differences on self-reported pain and fear, pulse or anticipation of the next BMA were found in relation to CBT versus general anaesthesia. The data collected in relation to parental stress and coping also demonstrated virtually no differences between CBT and general anaesthesia. However, parents rated significantly more behavioural adjustment symptoms at 24 hours after the BMA when their child had received anaesthesia. No differences were found in children's and parents' preference for CBT versus anaesthesia.

Kazak et al. ${ }^{59,60}$ (Table 2) used several of the same CBT components to compare the efficacy of conscious sedation with a combination of conscious sedation and psychological intervention for children undergoing BMA or LP. In a randomized, controlled prospective trial of 92 children with leukaemia, the combined treatment was found to be superior to conscious sedation alone on ratings of child distress by mothers and nurses. When contrasted with a cross-sectional control group consisting of the parents of 70 patients who were in first remission prior to the prospective study, the combined intervention group showed lower levels of child distress. The data also showed decreases in distress over time and concurrent improvements in quality of life and parental stress, and supported an inverse association between distress and child age.

Clearly, CBT has been shown in a number of studies to reduce the distress of children with cancer who are undergoing a variety of painful medical procedures and is considered a well- established treatment in the management of procedure-related pain.

Hypnosis Hypnosis is a term that is used in very different ways by different theorists, researchers and clinicians. Many attempts have been made to define it in terms of both the phenomena involved and the causal mechanisms, but no definition has yet satisfactorily answered all the questions raised by the subject matter. The term has so many different connotations (e.g. altered state, loss of will, enhanced abilities) that any attempt to define it is bound to have its drawbacks. The American Psychological Association, Division of Psychological Hypnosis (Division $30)^{61}$ defined hypnosis as:

a procedure during which a health care professional or researcher suggests that a client, patient, or subject experiences changes in sensation, perception, thought, or behaviour. The hypnotic context is generally established by an induction procedure. Although there are many different hypnotic inductions, most include suggestions for relaxation, calmness, and well-being. Instruction to imagine or think about pleasant experiences are also commonly included in hypnotic inductions. People respond to hypnosis in different ways. Some describe their experiences as an altered state of consciousness. Others describe hypnosis as a normal state of focused attention, in which they feel very calm and relaxed. Regardless of how and to what degree they respond, most people describe the experience as very pleasant. Some people are very responsive to hypnotic suggestion and others are less responsive... Hypnosis makes it easier for people to experience suggestions, but it does not force them to have those suggestions ...

The experience of procedure-related cancer pain has been the focus of numerous case reports and an increasing number of systematic studies in the hypnosis field (Table 3). In a classic study, Hilgard and LeBaron ${ }^{62}$ investigated the use of hypnosis in relieving pain and anxiety due to BMA in 24 children and adolescents with cancer. The patients ranged in age from six to 19 years and data were obtained through both self-report of pain and pain-related anxiety, and the observation of distress behaviour by an independent observer. Data were gathered both at baseline and post-hypnotic treatment times. For the 24 patients treated by hypnosis, statistically signifi- 


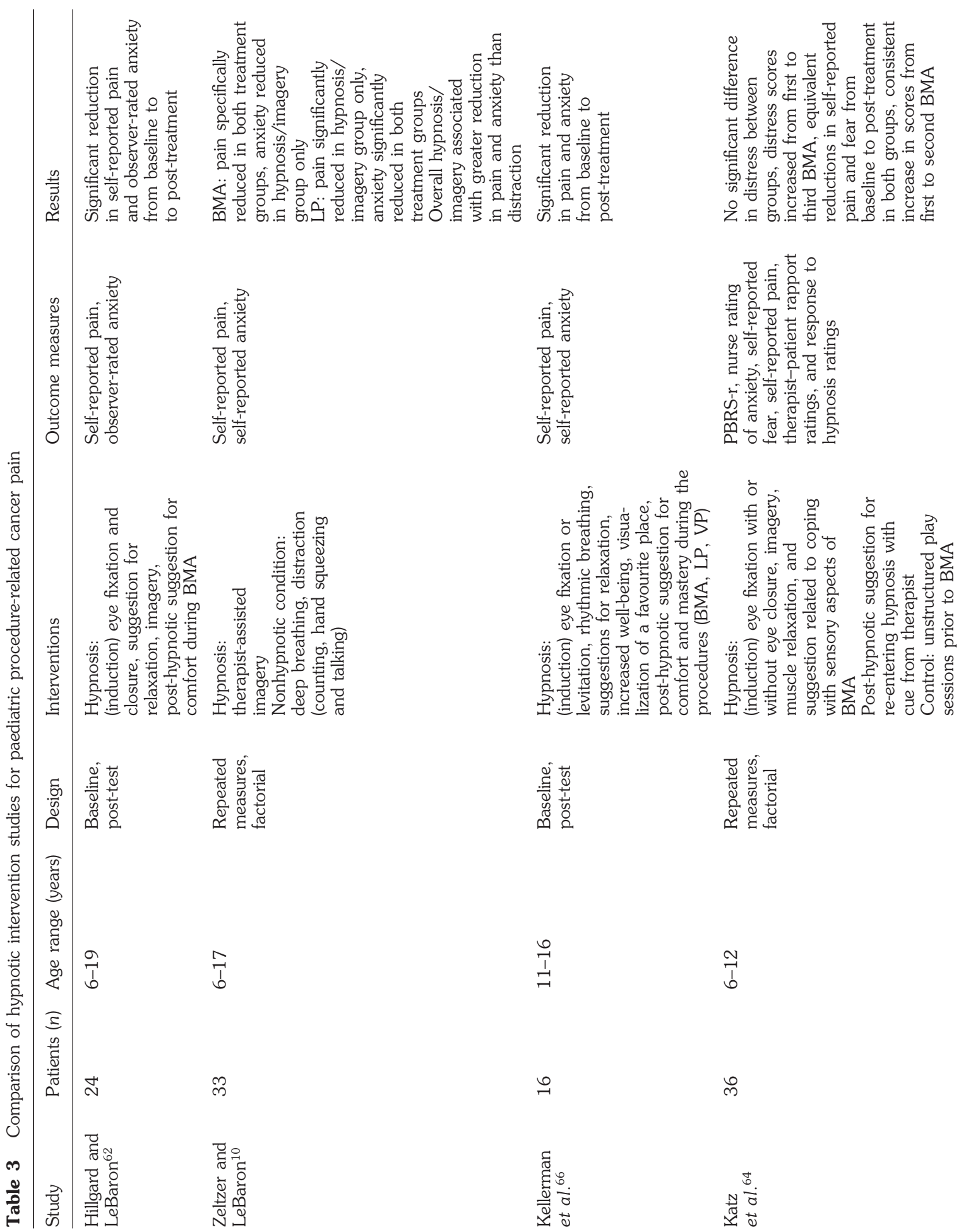



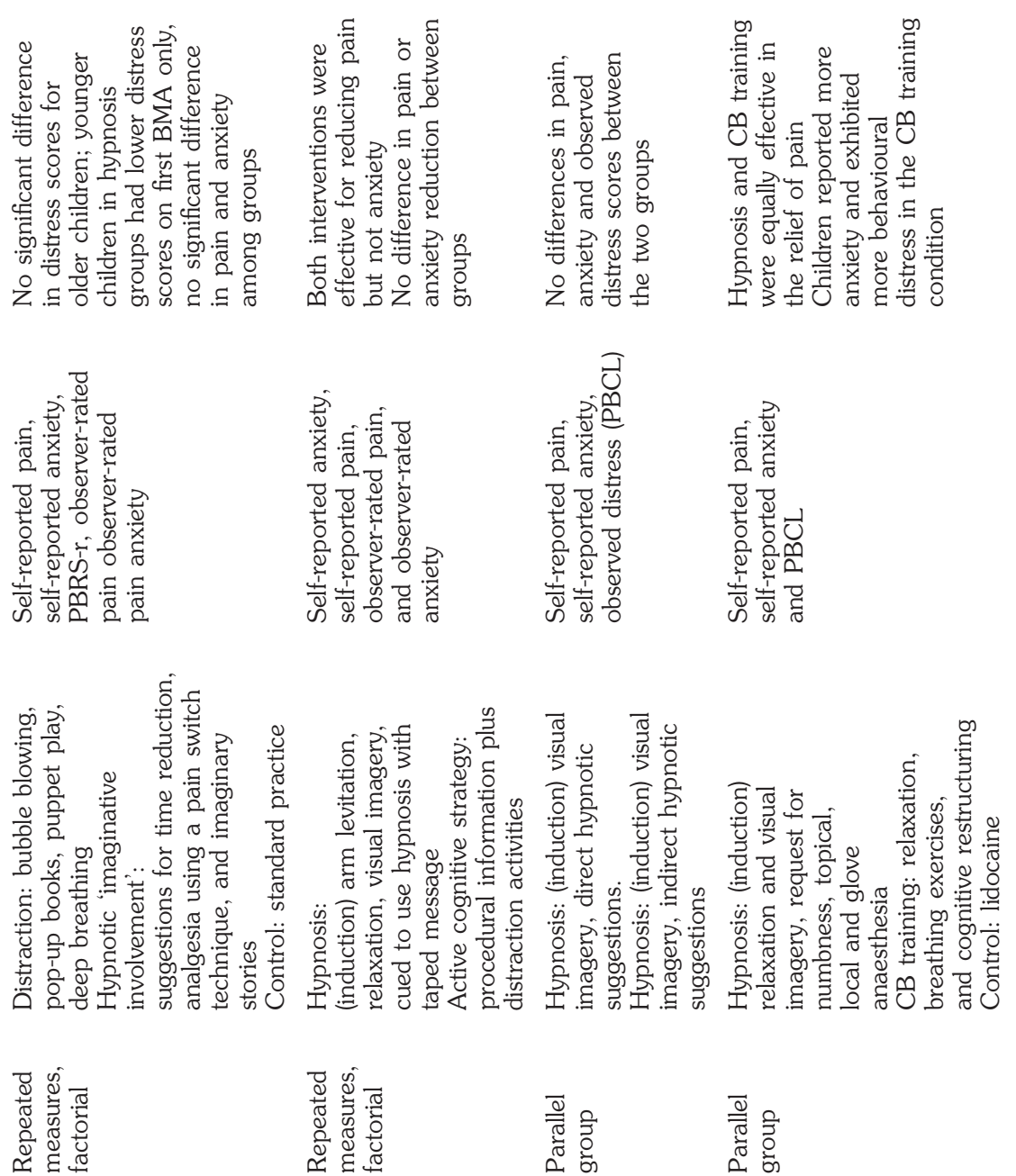

을

$\stackrel{\infty}{-1}$

$\frac{0}{1}$

ำ

กิ

ำ

ㅇ

요

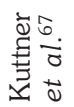

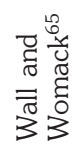

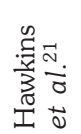

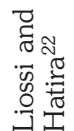


cant reductions over baseline occurred for both pain and anxiety in the first hypnotic treatment session, which were related significantly to hypnotizability as measured by the Stanford Hypnotic Clinical Scale for Children. ${ }^{63}$ Therapist attention, degree of rapport and amount of intervention were not controlled for in this study; these elements may have confounded the results. ${ }^{64,65}$

Zeltzer and LeBaron ${ }^{10}$ compared hypnotic with nonhypnotic behavioural techniques for efficacy in reducing pain and anxiety in 27 children and adolescents during BMA, and in 22 patients during LP. Forty-five patients consented to participate in the study; 12 (27\%) of those reported no need for intervention during the initial data gathering. The remaining 33 patients (aged 6-17 years) experienced considerable pain and anxiety during the procedures. Nonhypnotic behavioural techniques included a combination of deep breathing, distraction and practice sessions to help the child to control his or her fear. Both hypnotic and nonhypnotic interventions were conducted during the medical procedures, and patients in both groups were encouraged to have practice sessions. Both self-report and observational data were collected. For both LPs and BMAs, intervention was associated with an overall reduction in pain and anxiety. A significant interaction found between the amount of pain reduction and the type of intervention suggested that hypnosis was more effective than nonhypnotic techniques.

Kellerman et al. ${ }^{66}$ evaluated the effectiveness of hypnosis in reducing anxiety and discomfort in BMA, LP and chemotherapeutic injections in adolescents with cancer. Data were analysed for 16 patients (mean age 14 years). A small-sample design was used using the degree of change from baseline combined with multiple baselines across participants. Self-report measures were used as baseline data to assess the patient's anxiety and discomfort. Measures of anxiety and discomfort were recorded separately on five-point Likert scales $(1=$ none; $5=$ maximum $)$ and were gathered immediately before, during, and immediately after one of the above-mentioned procedures. Standardized psychological measures were also used to assess four dimensions: trait anxiety, self-esteem, health locus of control, and illness impact. Hypnotic interventions were individualized to the needs and interests of each patient. A significant reduction in both anxiety and discomfort at all three time periods were found after the hypnotic intervention. On the psychological measures, only a significant reduction in trait anxiety after hypnosis intervention was shown.

Katz et al. ${ }^{64}$ compared the effects of hypnosis with play comparison interventions in children undergoing repeated BMAs. Thirty-six children between the ages of six and 12 years (mean age was eight years three months) were randomly allocated to either a hypnosis or a play comparison group. Major components of the hypnotic intervention included the development of rapport, direct discussions about the child's medical history and treatment needs, active imagery tailored to the interests of each child, deep muscle relaxation, and suggestions. The specific suggestions incorporated were: imagery to reduce or reframe sensory/pain experiences; distraction and relaxation; pairing positive affect with medical procedures; developing a sense of mastery and control over sensory and affective experience; post-hypnotic suggestions for practising and reentering hypnosis at a cue from the therapist during actual procedures. The comparison study condition comprised nondirected play sessions that were designed to control for the amount of time and attention the child received from the psychologist who was performing the hypnotic intervention. Patients were followed for a period of six months after the psychological treatment. Immediately prior to the next three BMAs (after initial intervention), the children were seen in the clinic for a 20-minute intervention by the same therapist as they had encountered in previous sessions.

Both self-report and observer measures were utilized as dependent measures in this study. Children in both hypnosis and comparison groups demonstrated significant decreases in selfreport of fear and pain from baseline to postintervention BMA, with no major differences between groups. Thus, it appears that hypnosis and play are equally effective in reducing subjective pain and fear of BMA, while having no significant impact on observable behaviour, when group data are evaluated as a whole. 
Kuttner et al. ${ }^{67}$ compared distraction, a procedure-labelled hypnosis/imaginative involvement, and a standard practice control group, on the reduction of procedural pain and distress during BMA in 30 children, aged 3-10 years. In the distraction intervention, a therapist engaged the children in blowing bubbles, counting, puppet play and looking at pop-up books during the procedure. The hypnotic group received a combination of hypnotic suggestion, guided imagery, and therapist support. After treatment, each child was assessed during two later BMAs. The only significant finding to emerge indicated that, among younger children (three years to six years 11 months), the hypnotic treatment produced lower distress scores than did the distraction or control treatments for the first BMA only. By the second BMA, the younger children in the three groups showed equivalent reductions in distress scores. There were no significant differences in self-reported pain and anxiety among the three groups.

Wall and Womack ${ }^{65}$ examined the differential effects of standardized instruction in hypnosis or active cognitive strategy for the provision of relief from procedurally-induced pain and anxiety. In the active cognitive strategy group, patients were trained to use their own chosen distraction during the BMA or LP. The participants were 20 paediatric oncology outpatients ranging in age from five to 18 years. Baseline and postintervention data were obtained by both selfreport and observation measures. Interventions consisted of group practice sessions, where each group met twice in the week between baseline observations and post-intervention medical treatments. This study appears to be unique in its approach of using group treatment for cancer pain with children and adolescents. Experimenters were blind to the pre-intervention data. At the time of the second BMA or LP, patients were cued by audiotape to make use of the techniques learned during the training sessions.

The results indicated a significant treatment effect in the reduction of pain in both self-report and observed ratings. Anxiety, as rated by the patients' self-reports, was not reduced significantly. In examining differences in pain reduction by treatment, Wall and Womack ${ }^{65}$ found no significant differences between the hypnosis and the active cognitive strategies groups. The authors concluded that, although both techniques appeared to be significantly effective in pain reduction, neither was more effective than the other and neither appeared effective in anxiety reduction. In terms of hypnotizability, the authors reported no significant differences in results between high- and low-hypnotizable participants.

Hawkins et $a .^{21}$ examined the differential effectiveness of direct versus indirect hypnotic suggestions. Thirty children (age 5-15 years) with leukaemia and non-Hodgkin's lymphoma, who were undergoing regular LPs were randomly allocated to two groups. In one group, children were hypnotized and given direct suggestions associated with pain relief. Children in the second group were given indirect hypnotic suggestions (i.e. therapeutic stories and metaphors) associated with pain relief. After hypnotic intervention, there was a significant reduction over baseline for pain and anxiety during LP in both groups. Direct and indirect hypnotic methods were found to be equally effective. The study was performed in such a way that no conclusion regarding the level of analgesia was possible because no control group was used. However, the low pain scores indicated that effective analgesia was achieved during LP by both hypnotic interventions.

Liossi and Hatira ${ }^{22}$ conducted a randomized controlled trial to compare the efficacy of clinical hypnosis versus cognitive-behavioural (CB) training in alleviating the pain and distress of 30 paediatric cancer patients (age 5-15 years) who were undergoing BMA. Patients were randomized to one of three groups: hypnosis, a package of $\mathrm{CB}$ coping skills, and no intervention. In the hypnosis group children received hypnotic analgesic suggestions (i.e. request for numbness, topical, local and glove anaesthesia) and were given post-hypnotic suggestions. In the $\mathrm{CB}$ training group children were taught relaxation training, breathing exercises and cognitive restructuring. The control group, as the other groups, received a standard lidocaine injection. Outcome measures included self-reported pain, and anxiety and behavioural observation by an independent observer. The results demonstrated that patients who received either hypnosis or $\mathrm{CB}$ training 
reported less pain and pain-related anxiety than did the control patients, and less pain and anxiety than at their own baseline. Hypnosis and $\mathrm{CB}$ training were similarly effective in the relief of pain. The results also indicated that children reported more anxiety and exhibited more behavioural distress in the $\mathrm{CB}$ training group than in the hypnosis group.

To facilitate the comparison of the important elements of the studies reviewed, a table was devised, citing each study and providing a description of the participants, the research design, the major procedures, and the major findings.

Taken together, the results of these studies support the findings of laboratory pain studies with adults that individuals gain at least partial control over discomfort by using hypnosis. Hypnosis interventions were found to be of significant help in reducing pain and anxiety in all of the studies conducted so far. The consistency of the findings indicates the usefulness of hypnosis as an effective intervention for helping children and adolescents to control the pain and anxiety associated with medical procedures. Children enjoy the hypnotic experience. They obtain relief without destructive or unpleasant effects. There are no drug interactions, no reduction of normal function or mental capacity, and no development of tolerance to the hypnotic effect. A beneficial change in attitude towards cancer also fosters a sense of control. Moreover, hypnosis is an opportunity for the clinician to be inventive, spontaneous and playful, and to build a stronger therapeutic relationship with a child while providing pain relief.

Parents in pain treatment A particularly important aspect of preparing children for invasive procedures is the involvement of primary caregivers (normally parents or close relatives). There is a debate about whether parents should be present when children are undergoing such procedures. There is little doubt that children may be more disruptive if their parents are present. ${ }^{68,69}$ Observations of parent-child interactions during medical procedures suggest that parents may influence child distress by modelling distress behaviour, and/or that parents may differentially reinforce distress behaviours. For example, child distress has been observed to be greater when parents engage in behaviours that appear to communicate anxiety, such as agitation. ${ }^{70}$ Child distress has also been shown to be positively related to parental behaviours that pay attention to child distress behaviours, such as parental reassurance, ${ }^{70-74}$ explanation, ${ }^{74-76}$ giving control to the child (e.g. 'tell me when you are ready'), and criticism. $^{73}$

Almost all children want their parents to be present during painful procedures. ${ }^{18}$ Likewise, almost all parents would prefer to be present with their child at such a time. Most parents report that they are active in providing comfort and information to their children before and during the procedure. However, parents are not prepared for painful procedures. When they are trained, they can be of considerable assistance to their children. Parental use of distraction, relaxation and imagery appears to be associated with lower levels of child distress during general paediatric examinations, ${ }^{70}$ venepuncture, ${ }^{75,77}$ and BMA and LP procedures. ${ }^{59,73,78}$

The majority of parents are interested in learning simple interventions and they are willing to invest in the time to practise the techniques. The frequency of practice required by parents to acquire and maintain the necessary skill levels requires further investigation. Practice time and parental beliefs about the potential effectiveness of behavioural techniques needs to be considered in future investigations. ${ }^{78}$

Moreover, for parents themselves, invasive procedures are among the most traumatic aspects of treatment and these disturbing memories persist long after treatment ends. ${ }^{59}$ Jay and Elliott ${ }^{79}$ developed a Stress Inoculation Program for parents whose children were undergoing BMA or LP. This programme consisted of filmed modelling and education, self-statement training and relaxation, and was aimed at the parents' own personal stress associated with the medical procedures. Jay and Elliott found that the Stress Inoculation Program was helpful in reducing parental anxiety and in increasing their positive 'self-statements'. It is interestingly to note that they also found that parents who did not receive the Stress Inoculation intervention, and who simply observed their child's CB training, became as actively involved during the medical procedure, coaching the child to carry out breathing and 
imagery exercises, as did the parents who were in the Stress Inoculation Program. These results suggest that, while parental intervention is helpful, simply including the parents in the child's intervention can also have therapeutic consequences for them.

\section{How to treat children with cancer for procedure-related pain}

Although the majority of children who are diagnosed with cancer today will survive their illness, the medical treatment required to cure the disease remains extremely stressful. Most paediatric cancer patients receive repeated medical procedures over the course of several months or years. The resulting distress that these children experience during invasive medical procedures remains one of the most important quality of life issues in childhood cancer.

The use of conscious sedation or general anaesthesia for the first BMA, regardless of the child's age, and the use of conscious sedation for the LP, particularly for the preschool- or young schoolchild, is recommended. This optimal management of the initial experiences with these procedures prevents the child from developing extremely negative expectations of pain and distress for the repeated experiences that are part of many treatment protocols. The use of conscious sedation for subsequent BMAs, and possibly also LPs, combined with psychological interventions for most preschool and young school-age children, and maybe for older children and adolescents, is also recommended. However, some older school-age children and adolescents may prefer to use exclusively cognitive-behavioural and hypnotic pain management techniques in order to remain awake and alert during the procedure and minimize recovery time. ${ }^{31}$

The selection of both pharmacological and nonpharmacological interventions should be based on the factors identified as contributing to the pain and not based simply on the type of the procedure. It is critical to combine a 'state-of-theart' pharmacological protocol of analgesics and sedatives with psychological interventions to maximize the effective options that are available to patients and families. Several experimental studies indicate that offering patients a choice from several pain coping strategies results in greater pain reduction than just presenting one strategy.

In paediatric procedure-related pain and distress there is a gap between research knowledge and clinical practice. Although effective pharmacological and psychological interventions are available, few major institutions responsible for treating children with cancer have a systematic approach to pain management. It is recommended that such an approach should be based on three basic principles, namely, education, structure and emotional support (Figure 3). Professional education is mandated to provide adequate knowledge and skills training to produce competent practitioners in the management of procedure-related pain. As with all treatment, it is imperative that all patients and their parents should be adequately informed about the procedure and about the goals and limitations of the treatment strategy.

A clinical practice protocol is necessary to maximize the efficacy and safety of the interventions selected. Utilizing an approach based on a set of principles is also likely to facilitate the consistent application of the programme by the staff involved (i.e. to improve staff adherence to the programme), provided that the staff have been appropriately trained in the approach. It is necessary for children and their parents to know exactly what they are expected to do during the

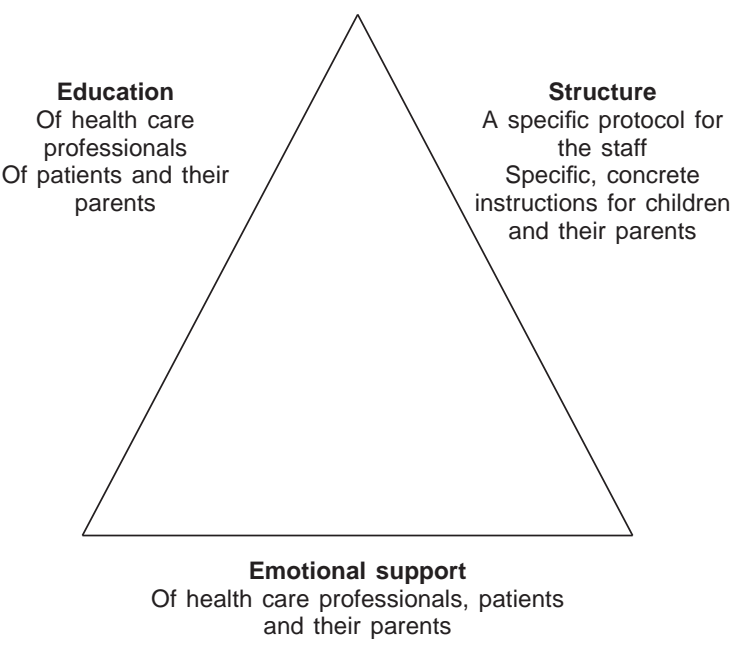

Figure 3 Basic principles for successful procedurerelated pain management 
procedure, so as to facilitate it and reduce their suffering. In general, structure increases efficiency and decreases distress, facilitates a smoother flow of procedures, and reduces the anxiety of health care professionals, and patients and their parents. Finally, all those involved in these medical procedures should receive continuous emotional suppport because of the extremely stressful nature of the experience.

\section{References}

1 Cancer Research Campaign. Childhood cancer - UK (Factsheet 15.1) London: CRC, 1990.

2 Stiller CA, Bunch KJ. Trends in survival for childhood cancer in Britain: diagnosed 1971-1985. Br J Cancer 1990; 62: 806-15.

3 Fowler-Kerry S. Adolescent oncology survivors' recollection of pain. In: Tyler DC, Krane EJ eds. Pediatric pain. Proceedings of the First International Pain Symposium, 1988 (Advances in pain research and therapy, vol. 15); Seattle. New York: Raven Press, 1990: 365-72.

4 Weekes DP, Savedra MC. Adolescent cancer: coping with treatment-related pain. J Pediatr Nurs 1988; 3: 318-28.

5 Kazak AE, Boyer BA, Brophy P et al. Parental perceptions of procedure-related distress and family adaptation in childhood leukemia. Child Health Care 1995; 24: 143-58.

6 Daut R1, Cleeland CS. The prevalence and severity of pain in cancer. Cancer 1982; 50: 1913-18.

7 Ljungman G, Kreuger A, Gordh T, Berg T, Sorensen S, Rawal N. Treatment of pain in pediatric oncology: a Swedish nationwide survey. Pain 1996; 68: $385-94$.

8 Miser AW, Dothage JA, Wesley RA, Miser JS. The prevalence of pain in a pediatric and young adult cancer population. Pain 1987; 29: 73-83.

9 Jay SM, Ozolins M, Elliott CH, Caldwell S. Assessment of children's distress during painful medical procedures. Health Psychol 1983; 2: 133-47.

10 Zeltzer L, LeBaron S. Hypnosis and non-hypnotic techniques for reduction of pain and anxiety during painful procedures in children and adolescents with cancer. J Pediatr 1982; 101: 1032-35.

11 Katz E, Kellerman J, Siegel S. Behavioural distress in children with cancer undergoing medical procedures: developmental considerations. J Consult Clin Psychol 1980; 48: 356-65.

12 Jay SM, Elliott C. Behavioral observation scales for measuring children's distress: the effects of increased methodological rigor. J Consult Clin Psychol 1984; 52: 1106-107.

13 McGrath P. Pain in children: nature, assessment and management. New York: Guilford Press, 1990.

14 McGrath PJ, Unruh AM. Pain in children and adolescents. Amsterdam: Elsevier, 1987.

15 Melzak R. The McGill pain questionnaire: major properties and scoring methods. Pain 1975; 1: 227-32.

16 Wong D, Baker C. Pain in children: comparison of assessment scales. Pediatr Nurs 1988; 6: 9-17.

17 Bieri D, Reeve RA, Champion GD et al. The Faces Pain Scale for the self assessment of the severity of pain experienced by children: development, initial validation and preliminary investigation for ratio scale properties. Pain 1990; 41: 139-50.

18 Ross D, Ross S. Childhood pain. Current issues, research and management. New York: Urban and Schwarzenberg, 1988.

19 LeBaron S, Zeltzer L. Assessment of acute pain and anxiety in children and adolescents by self-reports, observer reports, and a behavior checklist. J Consult Clin Psychol 1984; 52: 729-38.

20 Jay SM, Elliott CH. Behavioral observation scales for measuring children's distress: the effects of increased methodological rigor. J Consult Clin Psychol 1984; 52: 1106-107.

21 Hawkins PJ, Liossi C, Ewart B, Hatira P, Kosmidis H. Hypnosis in the alleviation of procedure related pain and distress in pediatric oncology patients. Contemp Hypnosis 1998; 15: 199-207.

22 Liossi C, Hatira P. Clinical hypnosis versus cognitive behavioral training for pain management with pediatric cancer patients undergoing bone marrow aspirations. Int J Clin Exp Hypnosis 1999; 47: 104-16.

23 Anand KJS. The applied physiology of pain. In: Anand KJS, McGrath PJ eds. Pain in the neonate. Amsterdam: Elsevier, 1993.

24 Kazak AE, Penati B, Waibel MK, Blackall GF. The Perception of Procedures Questionnaire: psychometric properties of a brief parent report measure of procedural distress. J Pediatr Psychol 1996; 21: 195-207.

25 Zeltzer LK, Jay SM, Fisher DM. The management of pain associated with pediatric procedures. Pediatr Clin North Am 1989; 36: 941-64.

26 Berde CB. Toxicity of local anesthetics in infants and children. J Pediatr 1993; 122: 14-20.

27 Burtin P, Ito S. Clinical pharmacology and toxicology of EMLA. In: Koren G ed. Eutectic mixture of local anesthetics (EMLA). A breakthrough in skin anesthesia. New York: Marcel Dekker, 1995.

28 Halperin DL, Koren G, Attias D et al. Topical skin anesthesia for venous, subcutaneous drug reservoir and lumbar punctures in children. Pediatrics 1989; 84: $281-84$.

29 Kapelushnik J, Koren G, Solh H, Greenberg M, DeVeber L. Evaluating the efficacy of EMLA in alleviating pain associated with lumbar puncture; 
comparison of open and double-blinded protocols in children. Pain 1990; 42: 31-34.

30 Calamandrei M, Messeri A, Busoni P, Bernini G, Lippi A, Tucci F. Comparison of two application techniques of EMLA and pain assessment in pediatric oncology patients. Reg Anesth 1996; 21: 557-60.

31 American Academy of Pediatrics Committee on Drugs. Guidelines for monitoring and management of pediatric patients during and after sedation for diagnostic and therapeutic procedures. Pediatrics 1992; 89: 1110-16.

32 Yaster M, Nichols DG, Deshpande J et al. Midazolam-fentanyl intravenous sedation in children: case report of respiratory arrest. Pediatrics 1990; 86: 463-67.

33 Sievers TD, Yee JD, Foley ME et al. Midazolam for conscious sedation during pediatric oncology procedures: safety and recovery parameters. Pediatrics 1999; 88: 1172-79.

34 Sandler ES, Weyman C, Conner K et al. Midazolam versus fentanyl as premedication for painful procedures in children with cancer. Pediatrics 1992; 89: $1110-16$.

35 Betcher DL, Burnham N. Midazolam for outpatient sedation. J Pediatr Oncol 1992; 9: 136-38.

36 Zeltzer L, Altman A, Cohen D, LeBaron S, Munuksela E, Schechter N. American Academy of Pediatrics Report of the Subcommittee on the Management of Pain Associated with Procedures in Children with Cancer. Pediatrics 1990; 86: 826-31.

37 Leahy S, Hockenberry-Eaton M, Sigler-Price K. Clinical management of pain in children with cancer. Cancer Pract 1994; 2: 37-45.

38 Forbes RB. General anesthesia for day care surgery patients. Can J Anesthesiol 1983; 63: 545-47.

39 Hain WR, Tomlinson JH, Barbor PR. Anesthesia for minor procedures in children with malignant disease. J R Soc Med 1985; 78: 715-20.

40 Fisher DM, Robinson S, Brett C, Perin G, Gregory G. Comparison of enflurane, halothane, and isoflurane for diagnostic and therapeutic procedures in children with malignancies. Anesthesiology 1985; 63: $647-50$.

41 Ferrari L, Barst S, Patila M, Bedford RF. Anesthesia for diagnostic and therapeutic procedures in pediatric outpatients. Am J Pediatr Oncol 1990; 12: 310-13.

42 Wark HJ. Post-operative jaundice in children: the influence of halothane. Anesthesia 1983; 38: 237-42.

43 Warner LO, Beach TP, Garvin JP, Weaver EJ. Halothane and children: the first quarter century. Anesth Analg 1984; 63: 838-40.

44 Jay SM, Elliott CH, Fitzgibbons I, Woody P, Siegel S. A comparative study of cognitive behavior therapy versus general anesthesia for children undergoing painful medical procedures Pain 1995; 62: 3-9.

45 Morton NS, Johnston G, White M, Marsh BJ. Propofol in pediatric anesthesia. Pediatr Anesth 1992; 2: 89-97.

46 Finley GA, MacManus B, Sampson SE, Fernandez CV, Retallick R. Delayed seizures following sedation with propofol. Can J Anesthesiol 1993; 40: 863-65.

47 Siegel LJ. Preparation of children for hospitalization: a selected review of the research literature. J Pediatr Psychol 1976; 1: 26-30.

48 Schechter NL. Pain and pain control in children. Curr Prob Pediatr 1985; 15: 1-67.

49 Anderson K, Masur FT. Psychological preparation for invasive medical and dental procedures. Behav Med 1983; 6: 1-8.

50 Neuhauser C, Amsterdam B, Hines P, Steward M. Children's concepts of healing: cognitive development and locus of control factors. Am J Orthopsychiatry 1978; 48: 325-41.

51 Lipowski ZJ. Physical illness, the individual, and the coping process. Psychol Med 1970; 1: 91-102.

52 Miller SM. Coping with impending stress: psychophysiological and cognitive correlates of choice. Psychophysiology 1979; 16: 572-81.

53 Jay SM. Invasive medical procedures. In: Routh D ed. Handbook of pediatric psychology. New York: Plenum Press, 1988: 401-25.

54 Turk DC, Meichenbaum D, Genest M. Pain and behavioral medicine: a cognitive behavioral perspective. New York: Guilford Press, 1983.

55 Meichenbaum DH. Cognitive behavior modification. New York, Plenum Press, 1997.

56 Jay SM, Elliott CH, Ozolins M, Pruitt C. Behavioural management of children's distress during painful medical procedures. Behav Res Ther 1985; 23: 513-20.

57 Jay SM, Elliott CH, Katz ER, Siegel SE. Cognitive behavioural and pharmacologic interventions for children undergoing painful medical procedures. $J$ Consult Clin Psychol 1987; 55: 860-65.

58 Jay SM, Elliott CH, Woody P, Siegel SE. An investigation of cognitive-behaviour therapy combined with oral valium for children undergoing painful medical procedures. Health Psychol 1991; 10: 317-22.

59 Kazak AE, Penati B, Brophy P, Himelstein B. Pharmacologic and psychologic interventions for procedural pain. Pediatrics 1998; 1: 59-66.

60 Kazak AE, Penati B, Boyer BA et al. A randomized controlled prospective study of a psychological and pharmacological intervention protocol for procedural distress in pediatric leukemia. J Pediatr Psychol 1996; 21: 615-31.

61 American Psychological Association. APA definition and description of hypnosis. Defining hypnosis for the public. Contemp Hypn 1994; 11: 142-43. 
62 Hilgard JR, LeBaron S. Relief of anxiety of pain in children and adolescents with cancer: quantitative measures and clinical observations. Int J Clin Exp Hypn 1982; 30: 417-42.

63 Morgan AH, Hilgard JR. The Stanford Hypnotic Clinical Scale for Children. Am J Clin Hypn 1978/79; 21(2 suppl 3): 148-69.

64 Katz ER, Kellerman J, Ellenberg L. Hypnosis in the reduction of acute pain and distress in children with cancer. J Pediatr Psychol 1987; 12: 379-94.

65 Wall VJ, Womack W. Hypnotic versus cognitive strategies for alleviation of procedural distress in pediatric oncology patients. Am J Clin Hypn 1989; 31: 181-91.

66 Kellerman J, Zeltzer L, Ellenberg L, Dash J. Hypnosis for the reduction of the acute pain and anxiety associated with medical procedures. $J$ Adolesc Health Care 1983; 4: 85-89.

67 Kuttner L, Bowman M, Teasdale M. Psychological treatment of distress, pain, and anxiety for young children with cancer. Dev Behav Pediatr 1988; 9: 374-81.

68 Gonzalez JC, Routh DK, Saab PG et al. Effects of parent presence on children's reactions to injections: behavioral, physiological, and subjective aspects. $J$ Pediatr Psychol 1989; 14: 449-62.

69 Shaw EG, Routh DK. Effects of mothers' presence on children's reaction to aversive procedures. $J$ Pediatr Psychol 1982; 7: 33-42.

70 Bush JP, Melamed BG, Sheras PL et al. Mother-child patterns of coping with anticipatory medical stress. Health Psychol 1986; 5: 137-57.

71 Blount RL, Powers SW, Cotter MW, Swan S, Free K. Making the system work. Training pediatric oncology patients to cope and their parents to coach them during BMA/LP procedures. Behav Modif 1994; 18: 6-31.

72 Blount RL, Corbin SM, Sturges JW, Wolfe VV, Prater JM, James LD. The relationship between adults' behavior and child coping and distress during BMA/LP procedures: a sequential analysis. Behav Ther 1989; 20: 585-601.

73 Blount RL, Sturges JW, Powers SW. Analysis of child and adult behavioral variation by phase of medical procedure. Behav Ther 1990; 21: 33-48.

74 Dahlquist LM, Power TG, Cox CN, Fernbach DJ. Parenting and child distress during cancer procedures: a multidimensional assessment. Child Health Care 1994; 23: 149-66.

75 Jacobsen P, Manne S, Gorfinkle K, Schorr O, Rapkin B, Redd B. Analysis of child and parent activity during painful medical procedures. Health Psychol 1990; 9: 559-76.

76 Manne S, Bakeman R, Jacobsen P. Adult-child interaction during invasive medical procedures. Health Psychol 1992; 11: 241-49.

77 Smith JT, Barabasz A, Barabasz M. Comparison of hypnosis and distraction in severely ill children undergoing painful medical procedures. J Couns Psychol 1996; 43: 187-95.

78 McCarthy AM, Cool VA, Petersen M, Bruene DA. Cognitive behavioral pain and anxiety interventions in pediatric oncology centers and bone marrow transplant units. J Pediatr Oncol Nurs 1996; 13(1): 3-14.

79 Jay SM, Elliott $\mathrm{CH}$. A stress inoculation program for parents whose children are undergoing painful procedures. J Cons Clin Psychol 1987; 58: 799-804. 
Reproduced with permission of the copyright owner. Further reproduction prohibited without permission. 